\title{
Article
}

\section{Practitioner perspectives of multi-agency safeguarding hubs (MASH)}

Shorrock, Sarah, Mcmanus, Michelle and Kirby, Stuart

Available at http://clok.uclan.ac.uk/31074/

Shorrock, Sarah, Mcmanus, Michelle and Kirby, Stuart ORCID: 0000-00023049-1248 (2019) Practitioner perspectives of multi-agency safeguarding hubs (MASH). The Journal of Adult Protection . ISSN 1466-8203

It is advisable to refer to the publisher's version if you intend to cite from the work. http://dx.doi.org/10.1108/JAP-06-2019-0021

For more information about UCLan's research in this area go to

http://www.uclan.ac.uk/researchgroups/ and search for < name of research Group>.

For information about Research generally at UCLan please go to http://www.uclan.ac.uk/research/

All outputs in CLoK are protected by Intellectual Property Rights law, including Copyright law. Copyright, IPR and Moral Rights for the works on this site are retained by the individual authors and/or other copyright owners. Terms and conditions for use of this material are defined in the policies page.

\section{CLoK}

Central Lancashire online Knowledge www.clok.uclan.ac.uk

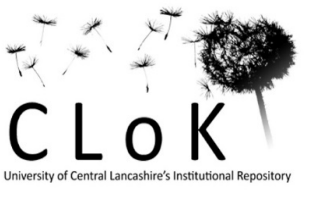




\section{Practitioner Perspectives of Multi-Agency Safeguarding Hubs (MASH).}

\begin{tabular}{|r|l|}
\hline Journal: & The Journal of Adult Protection \\
\hline Manuscript ID & JAP-06-2019-0021.R2 \\
\hline Manuscript Type: & Research Paper \\
\hline Keywords: & $\begin{array}{l}\text { Safeguarding, Multi Agency Safeguarding Hubs, Multi-Agency } \\
\text { Partnerships, Policing, Qualitative, Collaboration }\end{array}$ \\
\hline \multicolumn{2}{|l}{} \\
\hline
\end{tabular}

\section{SCHOLARONE \\ Manuscripts}




\title{
Practitioner Perspectives of Multi-Agency Safeguarding Hubs (MASH).
}

\begin{abstract}
Purpose - The challenges of transferring the theoretical requirements of an effective multiagency partnership into everyday practices is often overlooked, particularly within safeguarding practices. This paper, therefore, explores practitioner perspectives of working within a Multi-Agency Safeguarding Hub (MASH) and those factors that encourage or hinder a multi-agency approach to safeguarding vulnerable individuals.

Design/methodology/approach - Semi-structured interviews with 23 practitioners from one MASH location in the North of England were conducted, with a thematic analysis being used to analyse findings.
\end{abstract}

Findings - The interviews with practitioners illustrated the complexity of establishing a multiagency approach to safeguarding. It was inferred that whilst information sharing and trust between agencies had improved, the absence of a common governance structure, unified management system, formalisation of practices and procedures and shared pool of resources, limited the degree to which MASH could be considered a multi-agency approach to safeguarding.

Implications for Practice - Establishing a multi-agency approach to safeguarding is complex and does not occur automatically. Rather, the transition to collaborative practices needs to be planned, with agreed practices and processes implemented from the beginning and reviewed regularly.

Originality - Few studies have investigated the implementation of MASH into safeguarding practices, with this paper providing a unique insight into practitioner opinions regarding the transition to multi-agency practices. Whilst there is a focus on $\mathrm{MASH}$, the challenges to arise from the research may be reflective of other multi-agency partnerships, providing a foundation for best practice to emerge.

Keywords - Collaborative working, Multi-agency partnership, Multi-Agency Safeguarding Hub, Safeguarding 


\section{INTRODUCTION}

Serious case reviews have concluded that a lack of information sharing between agencies has resulted in vulnerable individuals being unnecessarily exposed to harmful or abusive situations (Preston-Shoot, 2017). In response to such criticisms, safeguarding policies and guidelines now advocate a need for safeguarding agencies to work more collaboratively, so that vulnerability can be identified and managed at the earliest opportunity (Care Act 2014). To reflect this move towards agency collaboration, Multi-Agency Safeguarding Hubs (MASH) were introduced in 2010, with the co-location of practitioners aiming to increase information sharing and joint decision-making (Home Office, 2014). The extent to which MASH has increased collaboration between safeguarding agencies has not been well documented, making it difficult to establish whether the recommendations of serious case reviews have been transferred into everyday practices. This paper explores the operational functioning of MASHs through practitioner experiences highlighting the reality of co-located safeguarding approaches to vulnerability.

\section{LITERATURE REVIEW}

Multi-agency partnerships have become a central feature of safeguarding practices, with serious case reviews, documents and policies outlining the benefits of agencies working collaboratively (Crawford \& L'Hoiry, 2015; Donnelly et al., 2017). The advantages of working across professional boundaries are associated with improved service delivery, better use of resources and reduced opportunities for vulnerable individuals to slip through the net (Fyson \& Kitson, 2012). Whilst such partnerships may be advantageous in terms of addressing complex public issues (Larkin \& Fox, 2009), research has demonstrated that achieving successful collaboration is difficult to implement (Stanley \& Humphreys, 2014). The establishment of an effective collaboration is often linked with an expectation for agencies and practitioners to seamlessly merge their organisational cultures and working practices. Such expectations can be problematic since safeguarding agencies generally develop cultures that reflect their role within society (Hatch \& Cunliffe, 2013), with practitioners often unwilling to move away from their traditional working practices (Crawford \& Evans, 2017). Other factors that may undermine the development of a successful partnership include an inability (or reluctance) to share information effectively, limited understandings of roles, 
differences in organisational priorities and thresholds and limited involvement of key agencies (McCreadie et al., 2012).

Successful partnerships depend upon the establishment of a clear division of labour and hierarchy of authority, with rules and procedures being jointly agreed upon, formalised and communicated to all practitioners (Jaques, 2017). Formalisation may include written rules, the development of handbooks or guidelines and distinct management systems (Walter et al., 2015). Too much formalisation can conflict with an individual's professional identity or judgement, potentially reducing the willingness of an agency or practitioner to engage (Sullivan \& Skelcher, 2017). Consequently, the formation of a multi-agency partnership does not occur instantly but requires planning and implementation in stages (Van Eyk \& Baum, 2002), with the development of trusting relationships enabling the dynamics of a partnership to change overtime (Piza et al., 2018).

Despite the challenges of designing and implementing multi-agency partnerships, collaborative working has become a central feature of safeguarding practices, with many UK police forces creating Multi-Agency Safeguarding Hubs (MASHs). The development of MASH has been influenced by the reoccurring theme of vulnerable children and young people being exposed to unnecessary abuse due to agencies not effectively working together and sharing information (Home Office, 2014; Dunne \& Finalay, 2016). Within England and Wales, a legal definition of MASH has not been established, some frameworks only process referrals relating to children and domestic abuse, whilst others include vulnerable adults (Shorrock et al., 2019). A core aim of most MASH frameworks is to improve safeguarding responses for children and vulnerable adults through information sharing, joint decision-making and coordinated interventions (Home Office, 2014). To ensure such principles are achieved, MASH co-locates a range of safeguarding agencies, mainly including the police, social services and health (King, 2012), allowing information to be shared in a secure environment. By co-locating agencies, MASH provides a single point of entry for concerns relating to individuals deemed 'at risk', enabling vulnerability to be identified and managed in a timely and appropriate manner (Allen \& Wilde, 2013).

To help assist with the processing of referrals, most MASH frameworks utilise a threestage approach (for more information see Shorrock et al., 2019). Stage one involves the identification of a vulnerable person and completion of a risk assessment (high, medium or 
standard), with the level of risk determining the speed at which a referral is processed. At the MASH site included in this research, the initial risk assessment was conducted by the police, but other MASH sites may have a different service completing the risk assessment or may have a different process to distinguish levels of concern. Referrals that meet the MASH criteria advance onto stage two, whereby agencies gather, share and analyse information relating to any referred individuals. If a referral does not meet the MASH criteria, it is often recorded as no further action and exists the process. The final stage of the process, known as the decisionmaking stage, involves a referral being jointly reviewed and relevant interventions being identified and implemented. Across MASH sites, the decision-making stage may be completed differently, with some sites only communicating via email, whilst others may have verbal, round table discussions.

Initial reviews indicate that MASH has started to move away from traditional silo working towards a multi-agency approach, with decisions being more informed, timely and dynamic (Allen \& Wilde, 2013; Crockett et al., 2013). Whilst such evaluations are informative, they often focus on frameworks that only process children and domestic abuse, overlooking adult safeguarding practices. Equally, practitioner experiences of transitioning from singleagency to multi-agency approaches to safeguarding has been overlooked by policy makers and researchers. Thus, this study focuses upon the daily experiences of MASH practitioners, examining the extent these reflect theoretical models of effective collaborative working in safeguarding individuals.

\section{METHODOLOGY}

\section{Data Source.}

A MASH site in the North of England was the focus of this research, with this MASH site being established since 2013. The site is spread over three locations, with two offices being located on local council premises and one located within a multi-purpose building that houses a range of businesses and services. Practitioners typically work from one office location, with the site being chosen due to the researchers' links with the local police force enabling data and practitioner access. Unlike most MASH sites, this MASH processed referrals relating to vulnerable children (aged $\leq 17$ ) and adults (aged $\geq 18$ or older), as well as domestic abuse (Government, (2016) definition), with all referrals made by the police. In a 12-month period, 
51,264 referrals were made, with $59 \%$ of these referrals relating to domestic abuse $(n=30,231)$. A fifth of referrals involved adults $(n=10,342,20 \%)$, whilst $21 \%$ of referrals related to children $(n=10,691)$. Agencies on the site included the police, child social services, adult services, probation, youth offending teams, health, mental health, education, children centres, fire and rescue and independent domestic violence advisors (IDVA). In total, 108 practitioners were associated with the MASH process, although not all 108 practitioners were permanently situated in one of the MASH offices. Most practitioners came from child social services ( $n=38,35 \%)$, with the police representing $31 \%$ of practitioners $(n=34)$, whilst adult social care accounted for $13 \%$ of all practitioners $(n=14)$. Nine practitioners came from Health $(8 \%)$, with 12 practitioners representing the other agencies $(n=11 \%)$. Only police practitioners process all referrals that are made, with practitioners from the other agencies only receiving and processing those referrals that involve individuals that reflect their service remit. For instance, all cases involving a child will be sent to child social services, with the police having the responsibility of sending referrals to relevant agencies.

To understand the daily workings of MASH, semi-structured interviews were conducted with $23 \mathrm{MASH}$ practitioners. Opportunity sampling was used to recruit practitioners, since shift patterns made it difficult to identify which practitioner would be available. To help recruit participants from a range of agencies, managers of all services represented in MASH sent out an email inviting practitioners to participate in a semistructured interview on a given day. Potential participants had the option of either registering for an interview prior to the interviews taking place or were asked on the day if they would like to participate. Participants came from the police $(n=8,35 \%)$, children social care $(n=6$, $26 \%)$, health $(n=4,17 \%)$, adult social care $(n=2,9 \%)$, probation $(n=2,9 \%)$ and education $(n=1$, $<1 \%)$. Of the 23 practitioners interviewed, five participants had managerial responsibilities (22\%), three participants were team leaders (13\%), with $65 \%$ of participants $(n=15)$ identifying themselves as referral or research assistants. All interviews were conducted on MASH premises, with participants required to sign a consent form prior to the interview commencing. Participants were informed that they could stop the interview at any time.

To help identify interview questions, a literature review examined the key strategies and theories relating to multi-agency working. Core principles of multi-agency working included effective recruitment and training, defined roles and responsibilities and strong 
communication strategies. The interview schedule reflected these identified principles, whilst allowing participants the opportunity to discuss other factors that contribute to a successful multi-agency partnership. To reduce interviewer variability, all interviewers were conducted by the same researcher and were recorded.

\section{Data Analysis}

To reflect the aims of this study, a positive epistemological approach was adopted, since the researchers were mainly interested in capturing practitioner understanding around what factors enable, as well as inhibit, the establishment of a successful multi-agency partnership. Therefore, data analysis was based upon what was observed, rather than the subjective opinions of the researchers. Through this approach, the researchers were able to hypothesise about the extent to which MASH can be considered a multi-agency approach, alongside highlighting effective and key issues in practice.

Once all interviews had been conducted and transcribed, a thematic analysis was undertaken following the principles identified by Braun and Clarke (2006). First, the researcher became familiar with the depth and breadth of the information gathered, with meanings and patterns being identified and recorded. The researcher then started to generate codes that related to important or interesting features of the data. In some instances, sections of texts were coded several times since they contained points which were interrelated and represented several themes. Interrater reliability analysis was run between the researcher and two academic colleagues, with an average association of $83 \%$ being identified. The third stage involved searching for themes within the coded data. Through this process, a series of candidate themes and sub-themes started to emerge, with all the data being assigned to one of the categories.

Next the researcher began to refine themes by merging similar candidate themes together, with candidate themes that had a lack of data support being removed. To aid with this process, Patton's (1990) dual criteria for judging categories was referred to. Data collated within each theme reflected a similar point (internal heterogeneity), whilst ensuring clear and identifiable differences across the individual themes (external heterogeneity). Five main themes were identified: Practices and Processes, Collaboration, Information Sharing and Joint Decision-Making, Availability of Resources and Impact of MASH. 


\section{FINDINGS}

\section{Practices and Processes.}

By providing one single referral point, MASH was supposed to help streamline routes of referrals for safeguarding agencies and the public. However, practitioners implied that referral pathways and thresholds were often inconsistent and not always multi-agency. At this site, only those referrals that had been referred by the police went through the MASH process, with referrals from other sources, such as social workers or education, being processed separately. For non-police referrals, the police did not contribute any information, contradicting the notion of MASH being a multi-agency approach to risk management.

"Usually, the Police go out to an incident and create a log... a [referral] is submitted...It is at that stage we receive a referral to start processing" (Police).

"It is hard to get any information [from the Police] ...They say, 'well it isn't safeguarding,' but the information they could give to us may lead to the safeguarding concern. We get a lot of anonymous referrals; a lot of allegations and we need to check with the Police to see if that has happened" (Child Social Care).

Processing times were also found to be inconsistent, with practitioners generally agreeing that the complexity of a case often dictated the amount of time needed to process it. For practitioners, processing times were determined by the number of risk factors present, the number of individuals needing to be researched and the availability of information.

"Depends upon the complexity of the case" (Police).

"A case can have anywhere between 1 and...16 people on... we check every adult on them against our databases...If we find that they are current to us, then we have more information to send back, so it takes a bit longer" (Probation Participant).

Several participants contradicted the notion that the complexity of a case influenced processing times. Instead, they argued that MASH had predetermined timeframes, which reflected the level of risk assigned to a referral.

"[Children's Social Care] have set timescales we work to. If it is an immediate safeguarding...it will be out in four-hours max...If we think there is a possibility it will 
meet the thresholds of Social Care interventions, we aim to process it in 24 hours. If it is likely to be early help or de-escalated, those would be 72 hours" (Child Social Care).

Participants felt that the processing of a MASH referral was also hindered by the way in which agencies identified risk. Practitioners generally worked from their agencies understanding of 'risk' and 'vulnerability', with the Police and Social Care defining 'risk' and 'vulnerability' differently, causing variances in thresholds.

"The Police are focused on crime and the criminal element. If a mother has been shoplifting, that could potentially be high-risk. For us [Children's Social Care], it is a factor, we would need to consider that. But it would certainly not be seen as high-risk for a child" (Child Social Care).

The absence of feedback may explain why practices and processes were inconsistent. Participants discussed that the contribution other agencies made to a referral, alongside the outcome of a case, was often unknown. Reasons to why practitioners were unaware of the contribution of other agencies, as well as the outcome of cases, was predominantly linked to information being stored on different databases. Once information had been provided and sent to the relevant agency, that referral was completed, and the next case was processed.

"I don't know... what happens at the very end, when it has been shared" (Police).

"There is that feedback for errors, but not really for the actual process" (Police).

Participants felt feedback regarding the outcome of a referral would be beneficial, since it would enable practices and processes to become more effective. For instance, feedback would indicate whether information shared was proportionate and necessary. Although, some did question the practicality of providing feedback on all cases processed, noting that demand and time constraints would make it difficult for practitioners to digest such feedback.

"It would be beneficial. We need to know...for job satisfaction. All you are doing is processing a queue... You don't know where it is going... You don't know if it is having an impact" (Police).

"No, not really. I wouldn't want to know that. It would be too much" (Child Social Care). 


\section{Collaboration.}

Despite inconsistencies in practices and processes, practitioners acknowledged that MASH had increased agency collaboration, with agencies collectively working together to safeguard vulnerable individuals at the earliest opportunity.

"Each agency... has an equal share and an equal say in safeguarding the people we deal with" (Police).

"The commitment is there strategically... [with all agencies] working to the same goal" (Health).

By working more collaboratively, practitioners mentioned that trust had started to develop between agencies and individuals, enabling practitioners to openly discuss safeguarding decisions and options with one another. Through these discussions, the role and responsibility of practitioners was communicated, enabling differences to be resolved. Practitioners felt confident about identifying these differences and using them productively:

"The Police's initial perspective is prevention and detection of crime and [Health] are looking at things from a slightly different angle. So generally, I think those discussions have been quite productive" (Health).

Participants felt that staff changes, which were quite frequent, hindered agency collaboration, since the introduction of new practitioners required the familiarisation process to start again. Rather than improving practices and processes, more time was spent on establishing working relationships.

"It is great when new people come in, because they have new ideas. They add new elements...But at the same time you get sick of having the same discussions... You go over the same stuff" (Police).

The temporary appointment of Police mangers and Health practitioners was also seen to reduce agency collaboration, since practitioners from these agencies often had an ulterior motive for joining the MASH team. For instance, to gain promotion within their service or to avoid other agency roles. Not only reducing continuity, but commitment. 
"I think the higher management tend to be temporary, as opposed to being permanent. I don't think they have the same vested interest as somebody if that was their substantive post" (Police).

Information Sharing and Joint Decision-Making.

Through an increase in agency collaboration, alongside co-location, practitioners had noticed an improvement to information sharing practices, with communication often occurring faceto-face, or via email. At the time of conducting the interviews, General Data Protection Regulation (GDPR) 2018 had not been introduced, therefore, reference to the implications these new regulations bring when storing and sharing information were not discussed. When information was shared, it generally related to an individual's demographic background and an overview of the agency's interaction with that individual. Questions were raised around the relevance of information, with the location of a practitioner influencing the quality of information shared.

"Inside the MASH it is verbal...Outside, to our Health partners, we task them by our electronic care system" (Health).

"Education, Health and Police are very consistent... The other agencies that aren't with us permanently, that is where differences in information emerges" (Child Social Care).

Information sharing agreements, agency protocols, acquirement of consent and practitioner's willingness to respond to information requests also influenced the sharing of information. Participants indicated that MASH had yet to establish a formal information sharing agreement, with the absence of this agreement making it difficult for practitioners to know what can be shared, with whom and how.

"The Adult team have a much tighter definition of what they can work with. So sometimes we don't always get the input from them that we might like to"'(Probation).

"Mental Health teams insist on consent. Unless there are factors that are a serious risk to that person... We can override that consent...[However,] how can it be true consent if we have concerns about...their capacity?" (Police).

Stemming from inconsistencies regarding the sharing of information, participants implied that joint decision-making within MASH was limited, with final decisions typically 
being made by either the Police or Social Care. The process of relying upon one agency to make a final safeguarding decision was criticised by several participants.

"We find all the information and send it back to the Police" (Probation).

"May be scope for [the final] decision to be more multi-agency...It's often a single agency decision" (Child Social Care).

Reasons for the lack of joint ownership related to processes, the presence of managers, access to Information and Communication Technology (ICT) databases and a need for someone to 'own' MASH.

"Children's Social Care... They have a lot more influence. I think that is because they have managers based within the department... We don't" (Police).

"Because the Police own the [MASH] system...there is a viewpoint, right or wrong, that the Police own MASH... We don't own MASH, the process, nor manage it. We tend to direct it... So, we do tend to lead on stuff" (Police).

Due to issues around information sharing, decision-making and ownership, numerous participants implied that silo working was still occurring. Whilst MASH may co-locate agencies, a multi-agency working environment had not been fully achieved.

"Despite the fact that we are co-located, we are not integrated. Still very much that the Police sit there, the Social Workers there...Still working in our own silos, with our own management, with our own agenda, with our own thresholds" (Police).

\section{Availability of Resources}

During the interviews, practitioners indicated that the demand placed on MASH often outweighed resources, reducing the capacity of MASH to process referrals in a timely and effective manner. The timeliness of safeguarding decisions was associated with the number of practitioners sat within the MASH, with practitioners often working at capacity due to their agency only providing one or two practitioners.

"It can be quite a big job. Especially if it has been a bank holiday weekend or one of us has had leave. [Probation] are looking at over a 100 [referrals] a day, and we do that each. So, if there is only one of us, you are looking at doing 200 plus" (Probation). 
"A lot of the delay there is around resources and having the capacity to do the work that is needed to be done...More resources would increase those timescales. We would have more room for better information" (Child Social Care).

The absence of a ICT database was associated with hindering the effectiveness of MASH, since various databases were used to store information. Access to these systems depended upon agency background and level of responsibility, causing the sharing of information to be complex. To improve the effectiveness of MASH it was suggested that all practitioners should use the same database when processing referrals.

"The administrative process... Is longwinded and time consuming. A lot of the steps are unnecessary...I think there needs to be more fluidity" (Police).

"It would increase workflow for us, because we would be cutting out the middle person" (Child Social Care).

Participants mentioned the lack of multi-agency training, with the level and type of training experienced by participants being inconsistent. For most participants, they "haven't received any (multi-agency) training" (Probation). If training had been experienced, it was either systems based, or simply shadowing colleagues and typically involved one agency.

"I got trained on the social care system...My training was to shadow really...In terms of formal training, a lot of it was just on the job" (Health).

Views regarding the necessity of training, either multi-agency or single-agency, were diverse, with opinions regarding the usefulness of specific MASH training being mixed. Rather, most participants referred to previous experiences of working in a safeguarding environment, with such roles providing them with the skills and professional judgement needed to process referrals.

"More training on [information sharing] ...To recap when to share" (Health).

"It would be very hard to teach...somebody who had started new. Never answered a phone call from the public or anybody...l think it is on the job training" (Police). 
Impact of MASH

Although participants identified and discussed various flaws within the current MASH framework, they did acknowledge two main benefits of introducing MASH into safeguarding practices. Practices and processes were now better than previous ones, and interagency collaboration had improved. Such benefits had enabled vulnerability to not only be identified earlier but managed more effectively.

"I think multi-agency working is always beneficial... We have been working alone in our different areas, we didn't know what was going on... So, it has got to be a beneficial thing and it does work" (Child Social Care).

"[MASH allows referrals] to come through a lot quicker than they used to. Before MASH, I would get [referrals] four months later and if there was a problem it was way too gone to do anything" (Adult Social Care).

\section{DISCUSSION}

The challenges of transferring the theoretical requirements of an effective multi-agency partnership into everyday practices is often overlooked, particularly within safeguarding practices. The introduction of MASH exemplifies this point, since the implementation of MASH into safeguarding practices has received little academic attention. To capture practitioner perspectives, semi-structured interviews were conducted with 23 MASH practitioners. Responses from these interviews illustrated that whilst MASH has taken a step towards enabling safeguarding agencies to work more collaboratively, full interagency collaboration has yet to be achieved.

For Cheminais' (2009), a multi-agency partnership involves practitioners from various agencies, working together to share information, tasks and responsibilities, so that risk can be identified and managed at the earliest opportunity. In support of this, the current study has highlighted that MASH has co-located a range of agencies into one central hub, enabling practitioners to routinely share information, make joint decisions and co-ordinate interventions (Home Office, 2013; 2014). Practitioners also acknowledged that trust between agencies had increased, with practitioners having a better understanding of agency roles and responsibilities, enabling practitioners to work towards the same goals. Subsequently, it 
seems that MASH has moved away from just being an organisational response to a complex issue and has started to influence working practices and processes (Horwath \& Morrison, 2007; Home Office, 2014).

The findings also reflect the notion that developing and achieving a successful multiagency partnership is complex and does not occur instantly but requires planning and commitment. For instance, it was found that practitioners within the MASH were responsible for specific tasks, such as processing referrals, sharing information, decision-making and overseeing daily practices. Such divisions of labour enabled practitioners to retain some of their professional identity, whilst allowing the overarching goal of MASH to be achieved. Conversely, the commitment of practitioners, specifically those at a management level, was seen to hinder the development of a collaborative working relationship, with the direction of MASH often changing with the appointment of a new manager, or strategic lead. Frequent managerial changes were seen to prevent MASH from moving forward, highlighting that whilst the role and responsibility of non-managerial practitioners was clearly defined, disjointed working practices occurred due to a lack of commitment and consistency at a management level.

The presence of numerous hierarchical structures was also associated with MASH not fully representing a collaborative approach to safeguarding, with practitioners indicating that ownership of MASH was not equally distributed amongst the various agencies involved. Instead of having one management structure, the activities of practitioners were directed by various agency managers, with managers often having a different understanding of how MASH should operate (Gano, 2003). The absence of a unified management system encouraged a single-agency decision-making culture to remain.

The degree to which joint decision-making had been embedded into practices was questionable, practitioners acknowledged that the sharing of information had become an integral part of the MASH process. By sharing information daily, alongside being co-located, practitioners recognised that safeguarding decisions were becoming more appropriate and proportionate, increasing the likelihood of interventions not only being implemented earlier, but also being more effective. The process of sharing information was not flawless, with practitioners suggesting that the usefulness of information shared was inconsistent and often influenced by the proximity of practitioners, information sharing agreements, agency 
protocols and issues regarding the acquirement of consent. Practitioners explained that gaining relevant information from Adult Social Care and Mental Health could be problematic, since these agencies were often located outside of the MASH office and typically required consent before sharing information. From an Adult Social Care and Mental Health perspective, legally (Health and Social Care Act (Regulated Activities) Regulations 2014) and ethically (Skills for Care, 2014) they are required to follow certain processes, particularly regarding consent. If MASH is to fulfil its intended purpose of identifying and managing risk at the earliest opportunity (Allen \& Wilde, 2013), agencies sat within MASH may need to spend time familiarising themselves with each other's information governance policies, so that reasons for withholding information can be better understood, without hindering decision-making process (Stevenson, 2014). Consequently, Doyle's (2008) conclusion that despite the necessity of co-location, collaboration does not automatically occur was evidenced, with the importance of introducing a degree of formalisation to practices and processes also being highlighted.

Throughout the research, the effectiveness and efficiency of MASH was associated with inconsistent practices and processes, with a lack of planning and agency agreement about the role, purpose and structure of MASH, contributing to this problem. The contribution of MASH agencies was a source conflict, since not all referrals received a multiagency response, with the source of a referral determining which agencies contributed to a case. For instance, it was noted that the police only processed those referrals that had been referred by the police, with referrals generated by the public, schools or other agencies, being processed without an input from the police. Conversely, health practitioners only provided information in relation to children. Thresholds relating to risk and vulnerability differed across agencies, with the police typically defining risk and vulnerability in terms of criminality, whilst social care adopted a victim perspective concentrating on vulnerability factors, such as living conditions. Such inconsistencies were reported to the identification and management of vulnerability at the earliest opportunity. This reiterates that whilst agencies may share the same goal of safeguarding vulnerable individuals, ways of achieving this goal are likely to differ (Gano, 2013; Home Office, 2014) and be influenced by legislative powers and agency responsibilities. To help promote successful collaboration therefore, the similarities and 
differences between agencies needs to be recognised understood and communicated, with definitions and thresholds becoming more consistent and formalised.

The availability of resources was seen to be another barrier preventing MASH from becoming more collaborative, with practitioners agreeing that the demands placed upon MASH often outweighed the availability of resources. The limited availability of time, resources and expertise further reduced the likelihood of MASH identifying and managing risk at the earliest opportunity. Within the literature it was inferred that insufficient operational and administrative staff (Burnett \& Appleton, 2004) and inconsistent agency contributions (Home Office, 2013) could prevent a seamless partnership from forming, with the findings of this study supporting these concerns. The findings of this research question the sustainability of MASH, since agencies may struggle to balance the demands placed upon MASH with the availability of resources. The lack of a standardised ICT system was perceived to be a major flaw within the MASH framework, with practitioners relying upon different databases to store, access and share information, preventing joint decision-making from occurring. The lack of an integrated ICT not only prevented a 'common language' from forming (Laming, 2003), but encouraged a single-agency culture to remain.

The absence of multi-agency training also limited the effectiveness of MASH, since practitioners had to rely upon their previous experience and willingness to learn on the job. Multi-agency training is deemed to be a crucial lever of joint working, with regular training enabling practitioners to feel valued, whilst allowing the various roles and responsibilities to be understood (Carpenter et al., 2010). By not incorporating regular multi-agency training into the MASH framework, practitioners acknowledged that a silo culture remained, with a lack of awareness around agency roles and responsibilities being associated with inconsistent processes. For some practitioners, an opportunity to learn about MASH systems, purpose and the role of other agencies would be beneficial and assist with the development of a multiagency culture. Other practitioners felt that MASH was not something somebody could be trained in, since MASH was a unique approach to safeguarding. These practitioners implied that learning on the job and relying upon previous experience was potentially more beneficial than a structured training programme. Demonstrating that whilst the benefits of multi-agency training have been widely documented, transferring these benefits into everyday working practices is complex and not always relevant for all multi-agency partnerships. 


\section{Implications for Practice}

Although this paper only focused upon the experiences of practitioners from one MASH location, the themes to emerge from these interviews have been found to resonate with other types of multi-agency partnerships or collaborative working environments (Golden et al., 2011; Home Office, 2014; Saunders, 2016). This paper has demonstrated that establishing an effective multi-agency partnership is complex, with all agencies and practitioners involved needing to move away from their traditional working cultures. Additionally, multi-agency partnerships need to ensure that information sharing, joint decision-making, clear management structures and relevant resources are not only embedded into practices and processes from the beginning but reviewed on a regular basis. To reinforce these findings, similar research needs to be conducted across other MASH locations and forms of multiagency partnerships. Research around what an effective and efficient integrated approach to safeguarding looks like across England and Wales is recommended, since vulnerability demand, population profiles, and availability/resources from services are different. Future research also needs to consider if and how a multi-agency approach to safeguarding results in better outcomes for vulnerable individuals compared to single agency approaches. Currently, there is a lack of knowledge around what agencies, processes and environments works best in achieving effective collaborative working that safeguards individuals. Detailed mapping of pathways and decision making of safeguarding referrals will undoubtedly help to identify best practice for effective MASH working, with cost effective and cost avoidance model work to be undertaken too.

\section{Conclusion}

By gathering the thoughts and opinions of MASH practitioners, this paper has demonstrated the challenges of transferring the theoretical principles of multi-agency partnership into every day working practices. It has highlighted that the co-location of agencies does not automatically result in an effective multi-agency approach to safeguarding. Rather, for a multi-agency partnership to be successful, consistent practices and processes need to be embedded into daily practices and regularly reviewed. To aid the transition towards a multiagency approach to safeguarding, key terms and thresholds, including risk and vulnerability, need to be standardised from the outset, with practitioners having a clear understanding of what information is needed to enable an informed decision to be made. Equally, to develop 
effective collaborative partnerships, a stable and consistent team needs to be established, with practitioners joining MASH for the right reasons, not just for promotion or short periods. To further enhance a sense of belonging and joint ownership, practitioners need opportunities to find out about each other's roles and responsibilities, as well as discuss the purpose of practices and processes, with feedback on cases and decisions, and the introduction of joint training providing an ideal starting point. Finally, to enable seamless collaborative practices, all practitioners need access to the same ICT systems, allowing decisions and processes to not only become more transparent, but assist in reducing the duplication of information. Thereby, decreasing processing times and increasing the likelihood of a safeguarding decision being made and implemented at the earliest opportunity. 


\section{References.}

Allen, C. \& Wilde, B. (2013), Birmingham Multi Agency Safeguarding Hub (MASH) Review, March 2013. Birmingham: Birmingham City Council.

Braun, V. \& Clarke, V. (2006), "Using Thematic Analysis in Psychology". Qualitative Research in Psychology, Vol. 3 No. 2, pp. 77-101.

Burnett, R. \& Appleton, C. (2004), “Joined-Up Services to Tackle Youth Crime A Case-Study in England". British Journal of Criminology, Vol. 44 No. 1, pp. 34-54.

Carpenter, J., Hackett, S., Patsios, D. \& Szilassy, E. (2010), Outcomes of Interagency Training to Safeguard Children: Final Report to the Department for Children, Schools and Families and the Department of Health. Bristol: Department for Children, Schools and Families.

Cheminais, R. (2009), Effective Multi-Agency Partnerships: Putting Every Child Matters into Practice. London: SAGE.

Crawford, A., \& Evans, K. (2017), "Crime Prevention and Community Safety", in Leibling, A., Maruna, S., \& McAra, L. (Eds.), The Oxford Handbook of Criminology (sixth edition), Oxford University Press, Oxford, pp. 797-824.

Crawford, A., \& L'Hoiry, X. (2015), “Partnerships in the Delivery of Policing and Safeguarding Children. An Exploratory Knowledge Platform for Policing: Exploiting Knowledge Assets, Utilising Data and Piloting Research Co-production", available at: https://essl.leeds.ac.uk/downloads/15/research and expertise (accessed 28 June 2019).

Crockett, R., Gilchrist, G., Davies, J., Henshall, A., Hoggart, L., Chandler, V., Simms, D., \& Webb, J. (December 2013), “Assessing the early impact of Multi Agency Safeguarding Hubs (MASH) in London: Final Report", available at: https://www.londonscb.gov.uk/wpcontent/uploads/2016/04/mash report final.pdf (accessed 28 June 2019). 
Donnelly, S., O'Brien, M., Walsh, J., McInerney, J., Campbell, J. \& Kodate, N. (May 2017), "Adult Safeguarding Legislation and Policy Rapid Realist Literature Review", available at: https://researchrepository.ucd.ie/handle/10197/9183 (accessed 28 June 2019).

Doyle, J. (2008), "Barriers and facilitators of multidisciplinary team working: a review", Nursing Children and Young People, Vol. 20 No. 2, pp. 26-29.

Dunne, J. F., \& Finalay, F. (2016), “G625 Multi-agency safeguarding hub-a new way of working", Archives of Disease in Childhood, Vol.101, pp. A369-A370.

Fyson, R. \& Kitson, D. (2012), “Outcomes following adult safeguarding alerts: a critical analysis of key factors", The Journal of Adult Protection, Vol. 14 No. (2), pp. 93-103.

Gano, D. L. (2003), Apollo Root Cause Analysis: A New Way of Thinking, Apollonian Publications, Washington.

Golden, S., Aston, H., \& Durbin, B. (2011), Devon Multi-Agency Safeguarding Hub: CaseStudy Report, NFER, Slough

Hatch, M. J., \& Cunliffe, A. L. (2013), Organization Theory: Modern, Symbolic and Postmodern Perspectives, Oxford University Press, Oxford.

Home Office (2013, July), “Multi-Agency Working and Information Sharing Project: Early Findings", available at:

https://assets.publishing.service.gov.uk/government/uploads/system/uploads/attachm ent data/file/225012/MASH Product.pdf (accessed 28 June 2019).

Home Office (2014, July), “Multi Agency Working and Information Sharing Project: Final Report", available at:

https://www.gov.uk/government/uploads/system/uploads/attachment data/file/3388 75/MASH.pdf (accessed 28 June 2019).

Horwath, J. \& Morrison, T. (2007), "Collaboration, Integration and Change in Children's Services: Critical Issues and Key Ingredients", Child Abuse and Neglect, Vol. 31 No. 1, pp. 55-69. 
Jaques, E. (2017), Requisite organization: A total system for effective managerial organization and managerial leadership for the 21st century, Routledge, London.

King, J. (2012), Local authority readiness assessment for MASH, London MASH Project Board, London.

Laming, W. (2003), The Victoria Climbié Inquiry-Report of an Inquiry by Lord Laming Presented to Parliament by the Secretary of State for Health and the Secretary of state for the Home Department by Command of Her Majesty, The Stationery Office, London.

Larkin, S. \& Fox, A. (2009), "Safeguarding policy and practice in Medway". The Journal of Adult Protection, Vol. 11 No. 3, pp.12-17.

McCreadie, C., Mathew, D., Filinson, R. \& Askham, J. (2008), “Ambiguity and cooperation in the implementation of adult protection policy", Social Policy \& Administration, Vol. 42 No. 3, pp.248-266.

Patton, M. Q. (1990), Qualitative Evaluation and Research Methods (2nd Edn), Sage, Beverly Hills, CA.

Piza, E. L., Kennedy, L. W., \& Caplan, J. M. (2018), “Facilitators and Impediments to Designing, Implementing, and Evaluating Risk-Based Policing Strategies Using Risk Terrain Modeling: Insights from a Multi-City Evaluation in the United States". European Journal on Criminal Policy and Research, Vol. 24 No. 4, pp. 489-513.

Preston-Shoot, M. (2017), "What Difference Does Legislation Make? Adult Safeguarding Through The Lens Of Serious Case Reviews And Safeguarding Adult Reviews", available at: http://ssab.safeguardingsomerset.org.uk/wpcontent/uploads/SW-SCRs-SARsReport-Final-Version-2017.pdf (accessed 28 June 2019).

Saunders, C. (2016), "Working together: building effective multi-agency partnerships", available at: http://www.police-foundation.org.uk/2017/wpcontent/uploads/2017/06/multi agency partnerships.pdf (accessed 28 June 2019). 
Shorrock, S., McManus, M. A., \& Kirby, S. (2019), "Investigating the Characteristics of Vulnerable Referrals Made to a Multi-Agency Safeguarding Hub", Policing: A Journal of Policy and Practice, Vol. 13 No.2, pp. 201-212.

Skill for Care. (2013), "Code of Conduct for Healthcare Support Workers and Adult Social Care Workers in England", Department of Health, available at: https://www.skillsforhealth.org.uk/images/services/code-of conduct/Code\%20of\%20Conduct\%20Healthcare\%20Support.pdf (accessed 6 September 2019).

Stanley, N. and Humphreys, C. (2014), "Multi-agency risk assessment and management for children and families experiencing domestic violence", Children and youth services review, Vol. 47, pp.78-85.

Stevenson, L. (2014), "Multi-Agency Safeguarding Hubs Improving Social Work Practice", available at: https://www.communitycare.co.uk/2014/07/30/multi-agencysafeguarding-hubs-improving-practice-finds-government-report/ (accessed 28 June 2019).

Sullivan, H., \& Skelcher, C. (2017), Working across boundaries: collaboration in public services. Macmillan International Higher Education.

Van Eyk, H. \& Baum, F. (2002), “Learning About Interagency Collaboration: Trialling Collaborative Projects Between Hospitals and Community Health Services", Health and Social Care in the Community, Vol. 10 No. 4, pp. 262-269.

Walter, S. G., Walter, A., \& Müller, D. (2015), “Formalization, Communication Quality, and Opportunistic Behavior In R \& D Alliances Between Competitors", Journal of Product Innovation Management, Vol. 32 No. 6, pp. 954-970. 